Cahiers de philosophie de l'université de

Totalitas. Aux origines d'un concept

\title{
Usages et concept de totalitas dans la psychologie thomasienne
}

\section{Maud Pouradier}

\section{(2) OpenEdition}

1 Journals

Édition électronique

URL : https://journals.openedition.org/cpuc/345

DOI : $10.4000 /$ cpuc.345

ISSN : 2677-6529

Éditeur

Presses universitaires de Caen

\section{Édition imprimée}

Date de publication : 31 décembre 2016

Pagination : 115-132

ISBN : 978-2-84133-842-9

ISSN : $1282-6545$

\section{Référence électronique}

Maud Pouradier, "Usages et concept de totalitas dans la psychologie thomasienne », Cahiers de philosophie de l'université de Caen [En ligne], 53 | 2016, mis en ligne le 01 février 2019, consulté le 03 février 2023. URL : http://journals.openedition.org/cpuc/345 ; DOI : https://doi.org/10.4000/cpuc.345

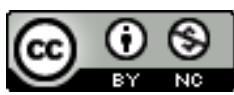

Creative Commons - Attribution - Pas d'Utilisation Commerciale 4.0 International - CC BY-NC 4.0 https://creativecommons.org/licenses/by-nc/4.0/ 


\section{Usages et concept de totalitas dans la psychologie thomasienne}

\section{$\mathrm{L}$}

E HOLENMÉRISME EST LE NOM DONNÉ par Henri More à la thèse selon laquelle il existe certaines entités qui peuvent être présentes tout entières à un tout et à chacune de ses parties. La thèse holenmériste suppose que l'entité en question demeure une: elle ne se multiplie ni ne se partitionne. L'argument holenmériste sert principalement à justifier l'ubiquité divine et la présence de lâme au corps, mais a également été utilisé dans la querelle des universaux et à propos de la présence des choses au temps ${ }^{1}$. Dans le second volume de L'Archéologie du sujet, Alain de Libera consacre une importante note au holenmérisme ${ }^{2}$. La formulation thomiste du holenmérisme dans la Somme de théologie ferait figure de texte topique en la matière: il synthétise la tripartition boécienne entre tout universel, tout intégral et tout potestatif, et les formulations augustiniennes du De Trinitate reprises dans le premier Livre des sentences. Je voudrais m'interroger sur le passage de la typologie boécienne du tout, à la typologie proprement thomiste, qui ne parle plus seulement des différentes sortes de tout, mais de totalité selon la quantité, l'essence ou la perfection (parfois la perfection de l'essence), auxquelles s'ajoute dans la plupart des textes (mais pas tous) la totalité selon la vertu ou selon la puissance.

Appelons thèse holenmériste stricte la thèse selon laquelle l'âme en tant qu'essence est tout entière présente à tout le corps et à chacune de ses parties. Si l'énoncé augustinien (tota anima est in toto corpore et in qualibet parte), assez flou, est largement accepté, il n'en va pas de même du holenmérisme strict, qui ne peut donc être élevé au rang de «thèse

1. J.-P. Anfray, «L'étendue spatiale et temporelle des esprits: Descartes et le holenmérisme», Revue philosophique de la France et de l'étranger, t. CXXXIX, nº 1, 2014, p. 23-46.

2. A. de Libera, Archéologie du sujet, t. II, La Quête de l'identité, Paris, Vrin, 2008, p. 438 sq. 
standard» de la scolastique ${ }^{3}$. En effet, la formulation augustinienne permet de soutenir que c'est sous deux modalités entièrement distinctes que l'âme est tout entière présente à tout le corps et à chacune de ses parties. C'est notamment le cas du Commentaire des sentences de Thomas d'Aquin: en tant qu'essence, elle est présente à chaque partie du corps qu'elle parfait; en tant que totalité de puissances, elle est présente tout entière à tout le corps. Si le terme de totalitas est bien utilisé ici par Thomas, il est manifestement synonyme d'universitas:

Et c'est pourquoi il faut dire avec Augustin que l'âme considérée selon son essence est toute dans chaque partie du corps. Et cependant non pas toute si l'on entend par là la totalité des puissances, car ainsi elle est toute dans tout l'être animét.

La différence est ici frappante avec la formulation du texte de la Somme de théologie:

Or, l'âme ne possède, ni par soi ni par accident, de totalité quantitative. Il suffit donc d'admettre qu'elle est toute dans n'importe quelle partie du corps, selon la totalité d'essence et de perfection; mais non pas selon la totalité de la vertus'.

Il est manifeste qu'ici totalitas ne peut à aucun titre être confondue avec universitas: on a affaire à un autre usage de cette notion, voire à un nouveau concept. L'objet de cet article est donc modeste: étudier la coélaboration de la thèse holenmériste stricte et d'un certain usage de la notion de totalité, et se demander si l'on peut en tirer un concept thomiste de totalité dans le domaine de la psychologie. Dans un premier temps, je m'intéresserai au matériau textuel initial de Thomas: la présentation de la formule augustinienne par le Lombard, et son commentaire par Albert le Grand. Je m'attacherai ensuite à la coélaboration du holenmérisme et de la

3. D. Des Chene, Life's Form. Late Aristotelian Conceptions of the Soul, Ithaca - Londres, Cornell University Press, 200o, p. 191 sq. L'auteur montre que derrière le «slogan» augustinien se cachent de multiples divergences, vidant ainsi de sa substance l'idée d'un holenmérisme «standard».

4. "Et ideo dicendum cum Augustino, quod anima secundum essentiam suam considerata, tota est in qualibet parte corporis. Non tamen tota, si accipiatur secundum totalitatem potentiarum; sic enim est tota in toto animali», Thomas d'Aquin, Scriptum super Sententiis, lib. 1 d. 8 q. 5 a. 3 co. Notre traduction. Les volumes 17 à 19 de l'édition léonine sont en préparation. On peut se reporter pour le texte latin au corpus thomisticum, http://www. corpusthomisticum.org/snp1008.html (page consultée le 24 mai 2016).

5. «Sed quia anima totalitatem quantitativam non habet, nec per se nec per accidens, ut dictum est; sufficit dicere quod anima tota est in qualibet parte corporis secundum totalitatem perfectionis et essentiae; non autem secundum totalitatem virtutis", Thomas d'Aquin, Somme théologique, t. I, A. Raulin (dir.), 2e éd., Paris, Cerf, 2011, Ia, qu. 76, art. 8, réponse, p. 676. Traduction légèrement modifiée. 
totalité par Thomas, en comparant le Commentaire des sentences, la Question disputée sur l'âme et la Question disputée sur les créatures spirituelles.

\section{L'âme est-elle tout entière dans tout le corps et dans n'importe laquelle de ses parties?}

\section{Fonction de l'adage augustinien}

Le texte mis en exergue par le Lombard au premier livre des Sentences est extrait du sixième livre du De Trinitate:

Si [l'âme] est plus simple que le corps, c'est parce qu'elle n'est pas matérielle, étendue dans l'espace, mais elle est dans chaque corps, toute dans le tout, toute en chaque partie. Aussi, qu'il se passe dans la plus petite partie du corps quelque chose que l'âme puisse enregistrer, bien que ce ne soit pas dans tout le corps, c'est toute elle qui le sent, parce que c'est à elle toute que cela apparait ${ }^{6}$.

Si l'âme sent tout entière ce qu'elle sent et est pleinement présente à elle-même en toute sensation et en tout sentiment, c'est parce qu'à son égard le concept de tout n'est pas adéquat. Aussi est-ce l'adjectif tota qui porte la thèse d'Augustin. La simplicité de l'âme est déduite de l'expérience de la présence à soi-même, qui atteste sous la forme de l'évidence que là où l'âme est, elle est tout entière. Qu'elle soit, par conséquent, aussi bien dans le corps entier que dans chacune de ses parties est moins une thèse qu'un constat: l'expérience de l'écart entre la circonscription locale de l'événement corporel et la plénitude totale du sentiment manifeste la présence du plus simple au plus complexe ${ }^{7}$. L'adage in toto tota et in qualibet ejus parte tota

6. "Nam ideo simplicior est corpore, quia non mole diffunditur per spatium loci, sed in unoquoque corpore, et in toto tota est, et in qualibet ejus parte tota est; et ideo cum fit aliquid in quavis exigua particula corporis quod sentiat anima, quamvis non fiat in toto corpore, illa tamen tota sentit, quia totam non latet», Augustin, La Trinité, t. I, Le Mystère (Livres I-VII), M. Mellet et T. Camelot (éd. et trad.), Paris, Desclée de Brouwer (Bibliothèque augustinienne, 15), 1955, VI, VI, $\$ 8$, p. 488-489. Traduction légèrement modifiée.

7. Augustin traite directement du problème de la présence de l'âme au corps dans le $D e$ animae quantitate, sections XIV-XXIII, in Dialogues philosophiques II, P. de Labriolle (éd. et trad.), Paris, Desclée de Brouwer (Bibliothèque augustinienne, 5), 1948, p. 272 sq. Sur l'influence plotinienne et porphyrienne de ces thèses augustiniennes: C. Couturier, "La structure métaphysique de l'homme d'après saint Augustin ", in Augustinus magister (Actes du congrès international augustinien, Paris, 21-24 septembre 1954), Paris, Études augustiniennes, 1954, t.I, p.543-550; J. Pépin, «Une nouvelle source de saint Augustin», Revue des études anciennes, n 66, 1964, p. 53-107; G. Madec, "La chair chrétienne": saint Augustin et la corporalité", Lectures augustiniennes, Paris, Institut d'études augustiniennes, 2001, p. 260-271. 
n'engage aucune thèse précise sur le type d'étant qu'est l'âme, mais nous invite seulement à penser une simplicité relative parmi les créatures.

La présentation des textes augustiniens par le Lombard dans le premier Livre des sentences n'articule pas cette présence à soi à une quelconque totalitas - terme qu'on ne trouve pas dans le Livre des sentences, qui ne comprend que l'adverbe totaliter ${ }^{8}$ - mais à l'aequalitas. Le maître des Sentences reprend l'idée d'une égalité des puissances de l'âme entre elles et chacune à elles toutes ${ }^{9}$ en la détachant de ce qu'on a appelé $a$ posteriori le «cogito augustinien ${ }^{10}$. L'aequalitas comme attribut de l'âme est l'infléchissement proprement lombardien de l'adage augustinien. Ni chez Augustin ni chez le Lombard nous ne sommes donc face à une thèse holenmériste.

Mais si l'âme n'est pas absolument simple, mais est à la fois simple et multiple, on ne peut pas en rester à l'idée selon laquelle l'âme est présente tota dans le tout et dans chaque partie. Comment comprendre que la simplicité relative de l'âme s'exprime par l'adjectif tota? Quel sens y a-t-il à parler de «toute l'âme» si elle n'a rien à voir avec un tout?

\section{Les outils du De divisione de Boèce}

À cette question on serait immédiatement tenté de répondre que c'est le concept boécien de "tout potestatif» qui permet à Thomas d'Aquin de résoudre cette tension. En effet dans la Somme de théologie, c'est la tripartition boécienne entre tout universel, tout intégral et tout potestatif qui est utilisée pour interpréter l'aeaqualitas de l'âme, tout en faisant de l'âme le sujet de ces puissances:

[L'essence de l'âme est-elle sa puissance?] Augustin parle ici de l'âme en tant qu'elle se connaît et s'aime elle-même. [...] Ou encore, comme certains le disent, cette manière [augustinienne] de parler est juste si l'on pense à la relation qu'un tout potestatif soutient avec ses parties. Le cas de ce tout est intermédiaire entre celui du tout universel et celui du tout intégral. [...] Le tout potentiel est bien présent en chaque partie avec toute l'essence, mais non avec sa puissance entière. On pourra donc en faire l'attribution à l'une

8. Thesaurus librorum sententiarum Petri Lombardi, J. Hamesse (éd.), Turnhout, Brepols, 1991.

9. Augustin, La Trinité, t. II, Livres VIII-XV, P. Agaësse (trad.), $2^{\mathrm{e}}$ éd., Paris, Institut d'études augustiniennes, 1997, X, XI, 18, p.154-156.

10. E. Bermon, Le Cogito dans la pensée de saint Augustin, Paris, Vrin, 2001. L’aequalitas devient d'autant plus une "propriété» de l'âme que le Lombard reformule les énoncés augustiniens concernant la connaissance de soi à la troisième personne. 
quelconque des parties, mais non aussi rigoureusement que dans le cas du tout universel ${ }^{11}$.

La réponse proprement thomiste à l'objection consiste à resituer la sentence augustinienne dans le contexte de son argumentation: alors que le Lombard détache l'aequalitas de l'âme dudit «cogito augustinien", Thomas la ressaisit dans le mouvement de pensée proprement augustinien. La seconde réponse, que Thomas ne considère manifestement pas comme pleinement suffisante (sicut quidam dicunt), s'appuie directement sur la tripartition boécienne topique:

En effet nous usons du terme de "tout» de multiples manières: ainsi le tout est ce qui est continu, comme le corps ou la ligne ou quelque chose du même genre; nous parlons aussi d'un tout à propos de ce qui n'est pas continu, comme le tout qu'est le troupeau, le peuple ou une armée; nous parlons aussi d'un tout à propos de ce qui est universel, comme Homme ou Cheval. En effet, ces derniers sont des touts de leurs parties, c'est-à-dire des hommes ou des chevaux, et c'est pourquoi nous appelons chaque homme particulier; on parle aussi d'un tout à propos de ce qui consiste en un certain nombre de vertus, comme la puissance rationnelle, la puissance sensitive et la puissance végétative de l'âme ${ }^{12}$.

Mais dans cet extrait du De divisione, la question n'est pas celle de la présence mais de la prédication. Exprimer l'aequalitas de l'âme, et $a$ fortiori une thèse holenmériste, serait donc particulièrement difficile dans le vocabulaire du De divisione. En effet les questions qui animent ce traité sont les suivantes: à quelles conditions peut-on prédiquer le tout de la partie? à quelles conditions doit-on dire que le tout a la partie? Le tout universel est prédicable de ses parties (on dit de l'homme qu'il est un animal, animal étant ici le tout universel ou générique dont les parties

11. «Augustinus loquitur de mente secundum quod noscit se et amat se. [...] Vel, sicut quidam dicunt, haec locutio verificatur secundum modum quo totum potestativum praedicatur de suis partibus, quod medium est inter totum universale et totum integrale. [...] Totum vero potentiale adest singulis partibus secundum totam suam essentiam, sed non secundum totam virtutem. Et ideo quodammodo potest praedicari de qualibet parte; sed non ita proprie sicut totum universale», Thomas d'Aquin, Somme théologique, t. I, A. Raulin (dir.), Paris, Cerf, 2011, Ia, qu. 77, art. 1, sol. 1, p. 678. Traduction légèrement modifiée.

12. "Quod enim dicimus totum multipliciter significamus: totum namque est quod continuum est, ut corpus vel linea vel aliquid hujusmodi; dicimus quoque totum quod continuum non est, ut totum gregem vel totum populum vel exercitum totum; dicimus quoque totum quod universale est, ut hominem vel equum, hi enim toti sunt suarum partium, id est hominum vel equorum, unde et particularem unumquemque hominem dicimus; dicitur quoque totum quod ex quibusdam virtutibus constat, ut animae alia potentia est sapiendi, alia sentiendi, alia vegetandi», Boèce, De Divisione Liber, J. Magee (éd.), Leyde - Boston - Cologne, Brill, 1998, 887d-888a, p. 38-43. Notre traduction. 
sont les espèces), ce qui n'est pas le cas du tout intégral. On ne peut pas dire que les briques sont la maison, en revanche, la maison a des briques. Le tout potestatif, qui intéresse plus directement la psychologie, apparaît comme un mixte du tout intégral et du tout universel, en ce qu'on peut prédiquer le tout de la partie (on parle d' «âme végétative», car on applique l'âme comme le genre à ses parties) bien que ces parties soient plutôt des parties intégrantes de l'âme d'un autre point de vue (puisque toutes les âmes n'ont pas la même structure, certaines possédant ou non la partie intellective, par exemple):

À propos du tout qui consiste en différentes vertus, sa division doit être faite de la manière suivante: «telle partie de l'âme est dans les plantes, telle autre dans les animaux", et à nouveau [en poursuivant la division] «en ce qui concerne l'âme des êtres animés, telle partie est rationnelle, telle autre est sensible» et celles-ci se partagent encore en de nouvelles divisions. Mais de ces choses l'âme ne constitue par leur genre mais leur tout [...]. De sorte que la division de cette sorte a quelque ressemblance et avec la division du genre et avec la division du tout, car en ce quelle que soit la partie de ce tout, s'ensuit sa prédication comme «âme», elle relève de la division du genre [...]; mais en ce que toute âme n'est pas constituée de toutes ces parties mais telle âme par telles parties, il est nécessaire que sa nature relève de celle du tout ${ }^{13}$.

Telle quelle, la typologie boécienne ne peut donc être appliquée pour exprimer la présence de l'âme à elle-même, encore moins la présence de l'âme au corps. C'est ici que le concept de totalitas devient utile. Lorsque dans ce même passage de la Somme de théologie, pour faire de l'âme le sujet de ses puissances, Thomas veut passer du problème de la prédication et de l'inhérence à celui de la présence de l'âme à ses parties (Totum vero potentiale adest singulis partibus secundum totam suam essentiam, sed non secundum totam virtutem), il introduit au sein de la tripartition boécienne une autre typologie, qu'il a déjà exposée dans la question précédente pour résoudre la question de la présence de l'âme au corps, et plus haut encore à propos de l'ubiquité de $\mathrm{Dieu}^{14}$, à savoir une typologie de la totalité selon l'essence ou selon la vertu. On ne saurait mieux souligner que la typologie

13. "Ejus quoque totius quod ex virtutibus constat hoc modo facienda est divisio: "animae alia pars est in virgultis, alia in animalibus", et rursus "ejus quae est in animalibus alia rationalis, alia sensibilis est" et rursus haec aliis sub divisionibus dissipantur. Sed non est anima horum genus sed totum [...]. Unde fit ut quiddam simile habeat hujusmodi divisio et generis et totius divisioni, nam quod quaelibet ejus pars fuerit animae praedicatio eam sequitur, ad generis divisionem refertur [...]; quod autem non omnis anima omnibus partibus jungitur sed alia aliis, hoc ad totius naturam referri necesse est ", Boèce, De Divisione Liber, J. Magee (éd.), Leyde - Boston - Cologne, Brill, 1998, 887d-888a, p. 38-43. Notre traduction.

14. Thomas d'Aquin, Somme théologique, t. I, Ia, qu. 8, art. 2 et 3, p. 202-205. 
de la totalité ne recouvre pas la typologie boécienne des touts, ni ne remplit la même fonction argumentative ${ }^{15}$.

\section{Deux concepts de totalitas dans le Commentaire des sentences d'Albert le Grand}

Thomas n'est pas le seul ni le premier à user de ce terme pour résoudre cette difficulté. Dans son propre Commentaire des sentences, Albert le Grand use du terme de totalitas pour reformuler l'égalité de l'âme à elle-même.

Il faut donc dire premièrement que l'âme possède une totalité selon de multiples sens, en un premier sens une totalité de forces, et en un deuxième sens qu'évoque Augustin au livre IX de la Trinité, en ce que ses attributs sont essentiels, comme le sont la vie, l'essence, la substance, et l'esprit. En ce sens en effet l'âme peut être toute en tout le corps, car en n'importe quelle partie il y a la vie, la substance, l'esprit, l'âme, le principe et la forme. Et selon tous ces attributs elle est dans l'être comme une certaine totalité. Il faut ajouter que le tout est la même chose que le parfait, et ainsi elle est toute en tout, et dans chaque partie. Si on parle de la totalité comme de l'ensemble des forces, elle serait toute en tout, mais pas dans chaque partie ${ }^{16}$.

Bien qu'Albert fasse fond principalement sur le concept de totum potestativum $^{17}$, il lui est nécessaire de sortir des divisions strictement boéciennes pour articuler la présence à soi-même de l'âme d'une part, et sa présence au

15. On a un procédé parfaitement similaire dans la Question disputée sur les créatures spirituelles, art. 11, réponse à la $2^{\text {nde }}$ objection, J.-B. Brenet (trad.), Paris, Vrin, 2010, p. 268-269.

16. "Dicendum ergo ad primum, quod anima habet multis rationibus totalitatem, scilicet quoad vires unam, et aliam quam tangit Augustinus in libro IX de Trinitate, quoad attributa sua essentialia, sicut sunt vita, essentia, substantia, et spiritus: quoad haec enim tota potest esse in toto corpore: quia in qualibet parte est vita, et substantia, et spiritus, et anima, et principium, et forma: et secundum omnia haec in esse est in ratione cujusdam totalitatis in esse. Dicitur etiam totum idem quod perfectum: et sic iterum tota in toto est, et in qualibet parte. Si autem dicitur totalitas ab universitate virium, erit in toto tota, sed non in qualibet parte tota", Albert le Grand, In I Sententiarum, Opera omnia, t. XXV, Paris, Borgnet, 1893, dist. 8, art. 26, p. 260. Nous traduisons.

17. Albert modifie le concept de «tout potestatif» au prisme de la hiérarchie de Denys. Le tout potestatif devient ainsi un tout intégratif, où le supérieur assume l'inférieur, de sorte que l'âme est pleinement présente aux fonctions supérieures sans l'être aux fonctions simplement végétatives et sensitives. Reformuler ainsi le "tout potestatif", c'est déjà permettre de penser la question de la présence au sein d'une catégorisation forgée pour s'interroger sur la prédication. Cependant, une telle reformulation ne peut suffire, en particulier pour penser la présence de l'âme à son corps. K. Park, «Albert's Influence on Late Medieval Psychology", in Albertus Magnus and the Sciences. Commemorative Essays, J. A. Weisheipl (éd.), Toronto, Pontifical Institute of Mediaeval Studies, 1980, p. 501-535; S. Cunningham, Reclaiming Moral Agency. The Moral Philosophy of Albert the Great, Washington DC, Catholic University of America Press, 2008, p. 125 sq. 
corps d'autre part. En tentant de justifier l'adage augustinien, Albert aboutit ainsi à deux acceptions de totalité: une totalité "pseudo-augustinienne", et qui doit caractériser l'égalité de l'âme lui permettant d'être toujours toute, et une totalité "pseudo-boécienne ", qui désigne le fait pour un tout d'être divisible, au moins en raison, en différentes parties, et qui n'ajoute au concept d'universitas que l'articulation en un tout structuré. Du point de vue de son essence, l'âme est une totalité pseudo-augustinienne, tandis que du point de vue de ses puissances, l'âme a une totalité. Dans un cas elle est une totalité, dans l'autre cas elle $a$ une totalité, qui n'est qu'un ensemble.

Commenté dans le cadre des Sentences de Pierre Lombard, l'adage augustinien est renversé : il ne s'agit plus, partant de l'expérience de la présence au corps, d'en déduire la simplicité relative de l'âme, mais sachant que l'âme est simple, d'expliquer comment elle peut être toute en tout le corps et en chacune de ses parties, sans elle-même être un tout qui se partitionne. Totalitas a donc pour fonction premièrement de résoudre la difficulté posée par le renversement de la question augustinienne, et deuxièmement de rendre compatible les divisions boéciennes du tout avec une interrogation sur la présence.

Or les acceptions pseudo-augustinienne et boécienne, bien que reposant sur le même terme, renvoient en réalité à deux concepts différents, que la médiation par l'idée de perfection ne permet pas d'unifier complètement. On ne sait d'ailleurs pas comment Albert comprend l'adage aristotélicien: faut-il comprendre que ce qui est parfait est tout, ou que le parfait est le tout? La première interprétation va dans le sens de la totalité pseudo-augustinienne tandis que la seconde va dans le sens de la totalité pseudo-boécienne. Cette ambiguïté donne ainsi lieu à une articulation difficultueuse, et non à une pleine unification conceptuelle.

\section{Élaboration thomiste du holenmérisme}

C'est donc au moment où un concept neuf surgit - le concept pseudoaugustinien - pour penser la présence totale de l'âme au corps, que ledit concept est immédiatement éclipsé par les divisions boéciennes. Or le tout potestatif boécien tend à limiter l'âme à une présence partielle au corps, en fonction de telle ou telle puissance. Mais lorsque la totalité échappe à la logique du tout (lorsqu'elle est une reformulation de l'aequalitas), elle permet certes de penser la présence, mais elle ne permet que de penser la présence à soi-même. Notre hypothèse est qu'une thèse comme ce qui a été appelé a posteriori le «holenmérisme» naît de la synthèse de ces deux concepts de totalité, synthèse permettant d'établir en quel sens identique l'âme est tout entière présente à tout le corps et à chacune de ses parties. 
Cette synthèse n'est pas définitionnelle mais fonctionnelle: il ne s'agit pas d'inventer un concept de totalité qui associerait de manière artificielle la signification du concept pseudo-augustinien de totalité à celle du concept pseudo-boécien de totalité, mais de concevoir la totalité de sorte qu'elle puisse assumer la double fonction de penser la présence, et la possibilité ou la non-possibilité de la partition. En effet, si la totalité ne permet pas de penser cette impossibilité de la partition, si elle est toujours pensée comme possibilité d'une division sur le modèle boécien, alors penser la présence de l'âme à tout le corps entraînera nécessairement sa répartition.

\section{Le Commentaire des sentences:}

\section{le rejet conjoint de la totalité et du holenmérisme}

Dans son Commentaire des sentences Thomas d'Aquin soutient que ce n'est pas selon le même sens que l'âme tout entière est présente à tout le corps et à toutes ses parties. En tant qu'essence ou forme substantielle, l'âme parfait chacune de ses parties et leur est donc tout entière présente. Il ne s'agit plus de décrire une expérience particulière, permettant de prouver la simplicité de l'âme. La présence totale de l'âme à chaque partie du corps est plutôt une conséquence de son statut de forme substantielle. Ainsi dans le sed contra, c'est parce que l'âme n'est pas présente dans le corps comme dans un lieu, mais comme la forme à la matière, qu'elle est présente tout entière en chacune de ses parties mais non à tout le corps. Dès lors, le corps est achevé par l'âme en un tout parce que l'âme n'est pas présente au tout du corps, mais à chacune de ses parties. Thomas prend ici une position rigoureusement symétrique à celle par exemple de Jean de la Rochelle, qui dans la Somme de l'âme affirmait au contraire qu'en tant qu'elle achève le corps, l'âme est présente à tout le corps mais à aucune de ses parties ${ }^{18}$. Au contraire, pour Thomas, la prise au sérieux du caractère substantiel de la forme invite au dépassement de la circonscription locale. En effet, si l'âme était présente au tout du corps, sa présence formelle serait comparable à celle d'un corps dans un lieu, et elle n'aurait pas la capacité d'achever le corps et de le parfaire. Elle serait une forme simplement accidentelle, circonscrite par ce qu'elle configure superficiellement. On arrive alors à une conclusion paradoxale: être forme substantielle du corps, c'est être l'acte

18. Jean de la Rochelle, De l'âme, J.-M. Vernier (trad.), Paris, Vrin, 2001, VI, chap. 42, p.111. En tant que l'âme parfait le corps «elle est dans le tout de telle sorte qu'elle ne soit en aucune partie: en effet, elle n'est pas la perfection d'une partie, mais du tout lui-même en tant que tel». 
de tout le corps et de chacune de ses parties, et être pleinement présent à chaque partie du corps mais non au tout du corps.

La référence au tout potestatif semble alors extérieure au mouvement de pensée thomasien, l'outil boécien ne servant qu'à sauver de justesse la totalité de l'adage augustinien. Mais son centre de gravité est inversé: ce qui était considéré comme une évidence (la présence totale de l'âme à tout le corps) devient difficultueux, tandis qu'est assurée par l'analyse hylémorphique la présence totale à chacune des parties. Dans cette reconfiguration hylémorphique de la question, Thomas ne peut affirmer une thèse proprement holenmériste, sauf à risquer de confondre forme substantielle et forme accidentelle. En effet, dans une perspective aristotélicienne comme dans une perspective boécienne, la totalité ne peut être rien d'autre que la manière dont se dit le tout, c'est-à-dire toujours un ensemble de parties ${ }^{19}$.

L'usage thomasien de l'expression secundum totalitatem, dans le passage du Commentaire des sentences cité en introduction, est ainsi notablement différent de celui d'Albert pour commenter la même sentence. Alors qu'Albert usait de l'expression secundum suam totalitatem pour désigner l'égalité de l'âme à elle-même et sa présence à soi, Thomas parle de l'âme tout entière secundum totalitatem potentiarum, où totalitas est manifestement synonyme d'universitas. En ce sens, dire que l'âme est «toute», ce n'est pas dire qu'elle est omniprésente à elle-même, mais dire qu'elle a toutes ses puissances. Elle est «toute» en tant qu'elle est le sujet de ses parties, qu'elle les a. Totalitas n'apporte ici manifestement aucune fonction nouvelle par rapport aux outils boéciens. On assiste plutôt, dans la réponse à la première objection, au rejet du concept de totalité, qui ne peut à aucun titre expliquer dans quelle mesure l'âme peut être «toute»:

Nous disons que l'âme est toute dans n'importe quelle partie du corps. Nous entendons par tout la perfection de sa nature, et non quelque totalité de parties; en effet le tout et le parfait, c'est la même chose, comme le dit le philosophe ${ }^{20}$.

19. Thomas d'Aquin, Sententia libri Metaphysicae, lib. 5, 1. 21, note 20. Le volume XLVI de l'édition léonine n'est pas encore paru. Pour le texte latin, on peut se reporter au corpus thomisticum: http://www.corpusthomisticum.org/cmpo5.html (page consultée le 24 mai 2016). Il existe une traduction française: Métaphysique d'Aristote / Commentaire de Thomas d'Aquin, t. I, G.-F. Delaporte (trad.), Paris, L'Harmattan, 2012.

20. "Dicimus totam animam esse in qualibet parte corporis, intelligimus per totum perfectionem naturae suae, et non aliquam totalitatem partium; totum enim et perfectum est idem, ut dicit philosophus», Thomas d'Aquin, Scriptum super Sententiis, lib. 1 d. 8 q. 5 a. 3 ad 1. Les volumes 17 à 19 de l'édition léonine sont en préparation. On peut se reporter pour le texte latin au corpus thomisticum, http://www.corpusthomisticum.org/snp10o8.html (page consultée le 24 mai 2016). Nous traduisons. 
L'adage aristotélicien ne sert pas, comme chez Albert, à tenter une synthèse entre deux concepts de totalité par l'idée de perfection, mais permet au contraire de faire de la totalité le simple synonyme d'ensemble (universitas), tandis que le tout peut être synonyme de parfait. Le sous-entendu ici est que le parfait est synonyme de ce qui est achevé non en ce qu'il aurait toutes ses parties, mais simplement en ce que rien ne lui manque ${ }^{21}$. Thomas se distingue alors de son maitre en refusant positivement la thèse strictement holenmériste pour mieux garantir le caractère substantiel de l'âme comme forme. C'est pourquoi c'est en tant que moteur que l'âme peut être considérée comme un tout potestatif, et in fine présente tout entière au tout du corps:

C'est pourquoi si l'on considère l'âme en tant que forme et essence, elle tout entière est en n'importe quelle partie du corps; mais si, en tant qu'elle est moteur, on la considère selon ses puissances, alors elle est tout entière dans tout le corps, et dans les diverses parties de celui-ci selon ses puissances ${ }^{22}$.

La thèse hylémorphique et l'approfondissement de la notion de forme substantielle permettent à Thomas de penser la question de la présence en se passant des outils augustiniens et d'une totalité pseudo-augustinienne. La difficulté est qu'en distinguant ainsi forme substantielle et forme accidentelle, l'adage augustinien est au fond moins justifié que mis à mal.

\section{La Question disputée sur l'âme: un usage boécien de totalitas?}

Contrairement au Commentaire des sentences, le concept de totalitas acquiert une fonction propre à partir de la Somme contre les gentils, mais je traiterai plutôt de la Question disputée sur l'âme, qui propose une typologie des totalités plus proche de la tripartition boécienne entre tout universel, tout intégral et tout potestatif. La plupart des auteurs qui ont tenté de reconstituer une histoire du holenmérisme ${ }^{23}$ citent la Somme de théologie, mais aussi l'article 1o de la Question disputée sur l'âme de Thomas d'Aquin. Or, si Thomas d'Aquin fait un usage spécifique de la totalité dans cet article 10,

21. «Un étant, en effet, est dit parfait dans la mesure où il est en acte, puisqu'on dit parfait l'être à qui rien ne fait défaut de sa perfection propre [secundum modum suae perfectionis]", Thomas d'Aquin, Somme théologique..., Ia q. 4 a. 1, réponse, p. 183. Dans la question 8 consacrée à l'ubiquité divine, cette perfection n'est pas associée au tout mais à la totalité.

22. "Unde si consideretur anima prout est forma et essentia, est in qualibet parte corporis tota; si autem prout est motor secundum potentias suas, sic est tota in toto, et in diversis partibus secundum diversas potentias", Thomas d'Aquin, Scriptum super Sententiis, lib. 1 d. 8 q. 5 a. 3 co. http://www.corpusthomisticum.org/snp10o8.html (page consultée le 24 mai 2016)

23. M. Rozemond, "Descartes, Mind-Body Union, and Holenmerism», Philosophical Topics, t. XXXI, nº 1-2, 2003, p.343-367. 
il continue de refuser une thèse holenmériste stricte. Comme dans le Commentaire des sentences, ce n'est pas selon la même signification que l'âme est présente tout entière au tout du corps et à ses parties. Pourtant, on assiste bien à l'établissement d'un usage spécifique de la totalité: cela s'explique par une différence d'accentuation. Alors que dans le Commentaire des sentences, l'acuité de Thomas d'Aquin porte plus particulièrement sur la présence à tout le corps et à toutes ses parties, dans la Question disputée sur l'âme, il s'intéresse spécifiquement à la manière dont l'âme peut être dite toute dans le premier et le second cas. Et dès lors il ne suffit plus de dire que l'âme peut être considérée comme forme d'une part et comme tout potestatif d'autre part: en tant que l'âme est forme substantielle du corps, dans quelle mesure peut-elle être toute présente au tout et à la partie? Autrement dit l'âme comme tout potestatif n'est plus une manière alternative de considérer l'âme, de sorte qu'on aurait affaire, comme dans le Commentaire des sentences, à une présence formelle d'une part et à une présence motrice d'autre part. Désormais la forme peut être considérée selon la totalité de la puissance d'une part et selon la totalité de la perfection de l'essence d'autre part. La totalité doit servir, au cœur même de l'analyse hylémorphique, à saisir la présence de l'âme au corps. Or cela modifie considérablement le sens qu'il faut donner au concept de totalité, car dans la Question disputée sur l'âme, l'âme comme forme substantielle n'apparaît comme un tout à aucun titre: en tant que forme simple, elle n'est pas composée de parties, et en tant que forme du corps, elle ne peut, seule, être achevée, bien qu'elle puisse être subsistante ${ }^{24}$. Seul l'homme tout entier, corps et âme, peut constituer un tout achevé. Dès lors la totalité n'est plus seulement la manière dont se dit le tout, mais aussi la manière dont ce qui n'est pas un tout peut se voir attribuer une totalité de sorte qu'on puisse dire que sa présence est totale. C'est le caractère total de ce qui n'est par essence que partiel, de sorte qu'il puisse avoir une présence totale et constituer un tout autre que lui. Ce faisant, Thomas trouve, du cœur de l'analyse hylémorphique, la nécessité de faire appel au concept néoplatonicien de totalité tel qu'il apparait dans la traduction des Éléments de théologie par Guillaume de Moerbeke ${ }^{25}$.

24. B. C. Bazan, «La corporalité selon saint Thomas», Revue philosophique de Louvain, t. LXXXI, $\mathrm{n}^{\circ}$ 51, 1983, p. 369-409; B. C. Bazan, «The Human Soul: Form and Substance?», Archives d'histoire doctrinale et littéraire du Moyen Âge, t. LXIV, 1997, p.95-126; A. C. Pegis, Saint Thomas and the Problem of the Soul in the Thirteenth Century, Toronto, St. Michael's College, 1934, où l'auteur montre que Thomas soutient une interprétation forte de l'adage augustinien dans ses œuvres de maturité contre son maître Albert le Grand dans son De anima.

25. Guillaume de Moerbeke, Proclus. Elementatio theologica, H. Boese (éd.), Leuven [Louvain], Leuven University Press, 1987, $\$ 69$. 
La réponse de l'article 10 de la Question disputée sur l'âme reprend et précise considérablement les analyses du Commentaire des sentences: pour la forme, être présente au corps, c'est être pleinement présente à chaque partie du corps, ce qui n'est rien d'autre que d'être forme de tout le corps et de constituer ainsi le corps comme tout. Pour reprendre l'expression de Bernardo Carlos Bazan, c'est parce que la présence de l'âme au corps est transcendantale, et non locale, que sa présence au corps semble défier les relations de tout et de partie ${ }^{26}$. On peut donc dire que l'âme est présente à tout le corps en ce qu'elle est forme du corps. De ce point de vue, la véritable présence de l'âme au corps consiste plutôt à être tout entière en chacune de ses parties, et seulement pour ainsi dire au tout. Mais le principal écart avec le Commentaire concerne la présence de l'âme selon ses puissances: non seulement il est précisé que c'est l'âme en tant que forme (et non en tant que moteur) qui peut être considérée sous l'angle de ses puissances, mais selon cette totalité, l'âme n'est présente ni au tout ni aux parties du corps.

Cette modification de la thèse thomiste est liée à la reformulation de l'idée de totalité. En l'occurrence, ce n'est plus selon la totalité des puissances mais selon la totalitas virtutis que la présence de l'âme au corps est interrogée. On assiste donc à une modification considérable de l'usage de totalité, qui n'est plus synonyme d'ensemble. On a comme une unification et une simplification de l'âme par le moyen terme de la totalité. Parler de l'âme comme d'un tout potestatif, c'est voir la possibilité de sa division pour faire de l'âme le sujet de ses puissances. Parler de l'âme selon la totalité de la vertu, c'est au contraire s'intéresser à l'âme dans l'unité de sa puissance plénière, tenter de conserver cette plénitude malgré la caractérisation de l'âme en termes de sujet. Aussi l'âme n'est-elle plus comme distribuée à travers le corps selon ses puissances, comme on pourrait en avoir l'impression en lisant le Commentaire des sentences. Au contraire, ce qui est mis en évidence est sa «transcendance» par rapport au corps, non au sens où elle ne serait pas forme du corps, mais en ce que l'âme humaine, par ce qu'elle a de plus propre (les opérations intellectuelles), montre une «indépendance opérative ${ }^{27}$. Ce n'est pas seulement une puissance parmi d'autres qui échappe au corps tout en l'informant, mais l'âme tout entière considérée selon la plénitude de sa puissance. On serait alors tenté de dire que, toutes proportions gardées, comme Dieu est présent au monde in totum sed non totaliter (c'est-à-dire selon toute son essence bien que son infinité actuelle en

26. B. C. Bazan, «La corporalité...», p. 373 .

27. Ibid., p.386. Pour les opérations intellectuelles, l'âme n'est pas seulement principe mais sujet. 
interdise une compréhension mondaine exhaustive ${ }^{28}$ ), selon sa puissance, l'âme est présente au corps tout entière mais non en totalité.

Comment Thomas a-t-il pu passer de l'idée d'une totalitas potentiarum à une totalitas secundum virtutem ou potentiam? Que signifie désormais la totalité pour permettre une telle unification de l'âme sous l'angle de sa puissance? Thomas reformule en termes de perfection de l'espace la totalité selon l'essence:

Nous disons donc que, puisque la perfection de l'espèce appartient à l'âme selon son essence, et que l'âme selon son essence est la forme du corps, et que selon qu'elle est forme du corps elle est en n'importe quelle partie du corps, comme il est montré, il reste que l'âme soit tout entière en n'importe quelle partie du corps selon la totalité de la perfection de l'espèce ${ }^{29}$.

Contrairement à l'homme tout entier, l'âme n'est pas composée de matière et de forme. En ce sens, on ne peut lui attribuer une «totalité selon l'essence». De même, stricto sensu, l'âme n'appartient pas à un genre et à une espèce: c'est l'homme qui possèderait une telle totalité selon l'essence. L'âme n'étant pas non plus achevée, contrairement à l'ange, on ne peut pas en toute rigueur lui attribuer une totalité selon la perfection:

Quelque chose est appelé un tout par rapport aux parties essentielles de l'espèce, comme la matière et la forme sont appelées parties du composé, et le genre et la différence d'une certaine manière parties de l'espèce: et ce mode de totalité est attribué aussi aux essences simples en raison de leur perfection, parce que, de même que les composées possèdent une espèce achevée par l'union des principes essentiels, de même les substances et les formes simples possèdent des espèces achevées par elles-mêmes ${ }^{30}$.

D'après la typologie proposée par Thomas, on ne devrait pas pouvoir parler de totalité à propos de l'âme quant à l'essence. Comment est-ce

28. A. Côté, L'Infinité divine dans la théologie médiévale. 1220-1255, Paris, Vrin, 2002.

29. "Dicimus ergo quod, cum perfectio speciei pertineat ad animam secundum suam essentiam, anima autem secundum suam essentiam est forma corporis et prout est forma corporis est in qualibet parte corporis, ut ostensum est, relinquitur quod anima tota sit in qualibet parte corporis secundum totalitatem perfectionis speciei», Thomas d'Aquin, Questions disputées. De l'âme, J.-M. Vernier (trad.), Paris, L'Harmattan, 2001, art. 10, réponse, p.156. Traduction légèrement modifiée.

30. "Alio modo dicitur aliquid totum per comparationem ad partes essentiales speciei; sicut materia et forma dicuntur partes compositi; genus et differentia partes quodammodo speciei. Et hic modus totalitatis attribuitur etiam essentiis simplicibus ratione suae perfectionis; eo quod sicut composita habent perfectam speciem ex coniunctione principiorum essentialium, ita substantiae et formae simplices habent perfectam speciem per seipsas ", ibid., p. 155 . Traduction légèrement modifiée. 
cependant possible? Il faut remarquer que parler d'une totalité selon la perfection, c'est ne plus lier directement la totalité à un mode de division. Certes, au début de la réponse, Thomas avait bien affirmé qu'il y a autant de totalités que de modes de partition d'un tout ${ }^{31}$. Deux interprétations sont donc possibles: ou bien on ne parle de totalité que métaphoriquement pour les choses simples, ou bien ce n'est que pour les substances composées que la totalité est adossée au mode de division, le cas de la forme simple achevée qu'est l'ange nous indiquant que la totalité peut s'entendre également de ce qui est simple. On a alors affaire à une totalité qui caractérise ce qui n'est pas un tout, mais est susceptible d'une présence totale.

C'est en ce dernier sens, si on le prend au sérieux, que la totalité peut être attribuée à l'âme selon une modalité inédite: la perfection de l'espèce peut s'appliquer à l'âme en ce que l'essence de l'âme en tant que forme du corps est d'achever le corps. Ici la perfection est actuation du corps: ce n'est qu'en ce sens seulement que la totalité de la perfection de l'espèce peut être attribuée à l'âme, et que selon cette totalité, elle est pleinement présente à chaque partie du corps - et non à son tout. La totalité ne désigne alors pas la possibilité de la division, mais plutôt ce qui, au cœur de ce qui peut n'être qu'une partie, permet l'achèvement du tout de l'homme, sans être la simple addition d'une ultime partie. Elle désigne ce qui a l'être du tout sans être le tout, ou encore elle est la totalisation même pouvant subsister sans achever actuellement le tout.

On a donc en toute rigueur deux idées de totalité: la totalité comme mode de la divisibilité, et la totalité comme mode de l'achèvement. Le statut particulier de l'âme est qu'elle peut être considérée selon le premier sens de la totalité, ou selon le second sens, si on la considère par rapport à ses puissances ou par rapport au fait qu'en tant que forme elle est perfection de l'espèce humaine. Il nous semble cependant que le second sens, soulignant le statut singulier de l'âme, prévaut sur le premier. Dans le cas contraire, il ne serait pas question de la «totalité selon la vertu», mais comme dans le Commentaire de la «totalité des puissances» de l'âme. Considérer la totalité de l'âme selon la puissance, ce n'est pas sérier ses différentes puissances, mais plutôt considérer la dimension intégrative du tout potestatif, tel qu'il est thématisé par Albert. Plus que la répartition des puissances dans les organes du corps, c'est plutôt la manière dont la puissance totale de l'âme transcende l'organicité du corps qui est soulignée.

31. «Potest autem attribui totalitas alicui formae tripliciter, secundum quod tribus modis convenit aliquid habere partes / Or la totalité peut être attribuée à une forme de trois manières, en ce qu'il convient à quelque chose d'avoir des parties selon trois modes», ibid., p. 154 . 


\section{La Question disputée sur les créatures spirituelles: un retour à Boèce?}

Par rapport à la Question disputée sur l'âme, l'article 4 de la Question disputée sur les créatures spirituelles est en retrait. Thomas semble revenir à une formulation plus classique de la typologie de la totalité:

Selon la totalité de l'essence, on peut absolument affirmer qu'elle est tout entière en chaque partie du corps, mais pas selon la totalité du pouvoir, parce que les parties sont parachevées par elle de différentes manières, en fonction d'opérations diverses, et qu'elle possède une opération, à savoir l'intelliger, qu'elle n'accomplit par aucune partie du corps ${ }^{32}$.

En effet dans la Question sur les créatures spirituelles, Thomas ne pense plus la présence totale de l'âme à ce dont elle est forme en fonction de la perfection, mais de l'acte: c'est à titre premier et par soi que l'âme est acte de tout le corps, et de manière secondaire qu'elle est l'acte de chaque partie du corps. C'est un point qui avait déjà été souligné par Thomas dans la première solution de la Question disputée sur l'âme; mais il est considérablement mis en valeur dans la réponse de la Question sur les créatures.

La modification la plus importante par rapport à la Question disputée sur l'âme touche la totalité selon l'essence. Dans la Question sur l'âme, était ouverte la possibilité de penser une totalité sans division. Dans la Question sur les créatures spirituelles, ces deux sous-divisions sont ramenées à une seule: il s'agit de la totalité selon la perfection de l'essence, qui peut concerner à la fois les substances composées et les substances simples. Elle implique une division entre forme et matière dans le premier cas, et une division simplement logique entre genre et espèce pour les formes simples comme l'âme. On perd donc la précision des formules de la Question sur l'âme. La thèse holenmériste est justifiée grâce à la distinction entre «à titre premier» et «à titre second»: c'est à titre premier qu'en tant qu'essence, l'âme est présente pleinement à tout le corps (l'âme est forme du corps) et à titre second aux parties du corps. L'idée de totalité tout à fait originale décelée dans la Question sur l'âme était-elle un hapax?

Je ne le crois pas, bien qu'incontestablement cette précision ne soit pas soulignée par Thomas dans la Question sur les créatures spirituelles, du fait de l'objet plus large qui occupe le docteur angélique. Nous avons un indice

32. "Secundum totalitatem essentie simpliciter enuntiari possit esse tota in qualibet corporis parte, non autem secundum totalitatem virtutis, quia partes difformiter perficiuntur ab ipsa ad diversas operationes, et aliqua oepratio est eius, scilicet intelligere, quam per nullam partem corporis exequitur », Thomas d'Aquin, Les Créatures spirituelles, J.-B. Brenet (trad.), Paris, Vrin, 2010, art. 4, réponse, p.138-139. 
que cette précision demeure effective dans la réponse à la dix-septième objection de ce même article:

[Objection.] 17. Puisque l'âme est simple et dépourvue de quantité dimensionnelle, il semble qu'on ne puisse lui attribuer aucune autre totalité que la totalité relative au pouvoir; or elle n'est pas dans chaque partie du corps selon ses puissances, où l'on place la totalité de son pouvoir; donc l'âme n'est pas tout entière dans chaque partie du corps. [...]

[Réponse.] 17. L'âme étant indivisible, il s'ensuit qu'elle n'a pas la totalité quantitative; mais cela n'implique pas pour autant qu'on ne trouve en elle que la totalité des puissances: il y a en elle, en effet, la totalité relative à la notion de l'essence, ainsi qu'on l'a dit ${ }^{33}$.

Le sens obvie de cette réponse consiste évidemment à renvoyer l'objecteur à la tripartition de la totalité citée plus haut. Mais la réponse porte également sur le sens qu'il faut attribuer au mot de totalitas: dans la formulation de l'objecteur, la totalitas virtutis ne correspond à rien d'autre qu'à la totalitas potentiarum, ce qui revient à dire que parler de totalité selon la vertu revient à parler de tout potestatif. La réponse de Thomas remet en cause cette identification entre la totalité selon le pouvoir et le tout potestatif. Si les deux choses ne sont pas équivalentes - autrement dit si la totalitas virtutis n'est pas égale à la totalitas potentiarum, et par conséquent si totalitas n'est à aucun titre synonyme d'universitas - c'est précisément parce que la prise au sérieux de l'âme comme forme permet d'aller au-delà de l'analyse de l'âme en termes de tout potestatif, et de considérer l'âme selon son excellence (virtus), laquelle assume toutes les puissances dans l'unité. Le renvoi à la totalité selon l'essence est donc un renvoi à l'essence de l'âme en tant que forme substantielle, laquelle permet - contrairement aux divisions boéciennes - de considérer l'âme selon la totalité de sa vertu (qui doit être entendue aussi bien au sens de puissance que d'excellence). Le concept de totalité demeure donc moins adossé à la divisibilité qu’à la simplicité ${ }^{34}$. Il renvoie à l'instance de totalisation subsistante qu'est l'âme,

33. "17. Cum anima sit simplex et absque quantitate dimensiva, nulla totalitas videtur ei posse attribui nisi virtutis; set non est in qualibet parte corporis secundum suas potentias, in quibus consideratur totalitas virtutis eius; non ergo in qualibet parte corporis est tota anima. [...] 17. Ad septimum decimum dicendum quod anima, ex hoc quod est indivisibilis, sequitur quod non habeat totalitatem quantitatis; nec proter hoc relinquitur quod sit in ea sola totalitas potentiarum: est enim in ea totalitas secundum essentie rationem, ut dictum est », ibid., p. 132-133 et p. 142-143.

34. Nous soutenons donc une hypothèse contraire à la série de quatre articles de B. Bro dans la Revue thomiste - ce qui ne signifie pas que ces textes n'ont pas été une référence essentielle pour ce travail. B. Bro, «La notion métaphysique de tout et son application au problème 
bien qu'elle ne soit elle-même ni un tout ni pleinement totalisable (au moins selon la puissance).

Comment articuler dès lors la totalité comme simple mode d'un tout divisible, et la totalisation subsistante qui n'est pas un tout et se caractérise par sa simplicité? Est-ce possible, et ne faut-il pas voir tout simplement un usage lâche du terme de totalité quant à l'âme humaine? On avait chez Albert la difficulté d'articuler une totalité pseudo-augustinienne et une totalité pseudo-boécienne. Il me semble qu'on a affaire chez Thomas à un concept aristotélicien de la totalité d'une part, et à un concept néoplatonicien d'autre part. Le premier renvoie à la totalité comme l'unité de ce qui est divisible, le second renvoie non seulement à la présence du tout, mais dans le cas de l'âme à l'instance de totalisation qui n'est elle-même pas pleinement totalisable (en tout cas selon la puissance). Comme chez Albert, c'est le concept de perfection qui doit permettre d'unifier ces deux acceptions de la totalité: la perfection du composé est d'être le tout de ses parties, et la perfection du simple d'être en acte. Si la perfection de l'âme est de former actuellement le corps, elle est cependant la perfection subsistante de ce corps, de sorte qu'elle puisse elle aussi constituer une totalité. Au cœur de l'analyse hylémorphique, et alors qu'il use des concepts aristotéliciens, Thomas recourt pour des raisons argumentatives à un concept qui, fondamentalement, est d'inspiration néoplatonicienne, pour répondre à une question qui n'est ni celle de la prédication ni celle de l'inhérence, mais celle de la présence. Le holenmérisme naît de cette rencontre entre une interrogation d'inspiration néoplatonicienne et un hylémorphisme aristotélicien. C'est pourquoi le concept de totalité, tel que nous l'avons caractérisé, est intrinsèquement lié à son élaboration.

Maud Pouradier

Normandie Univ, UNICAEN, Identité et Subjectivité (EA 2129), 14000 Caen, France

théologique de l'union hypostatique", Revue thomiste, n 67, 1967, p. 29-62, et nº 68, 1968, p.181-197, p. 353-38o, p. 561-83. 